\title{
Controlling aero-elastic instability of curtain wall systems in high-rise buildings
}

\author{
$\underline{\text { B. H. Tan }}^{\text {a }}$, A. D. Lucey ${ }^{\text {a }}$ and M. W. Pitman ${ }^{a}$ \\ ${ }^{a}$ Fluid Dynamics Research Group, Curtin University GPO Box U1987, Perth, WA 6845, Australia \\ Email: benhoea.tan@postgrad.curtin.edu.au
}

\begin{abstract}
Nowadays modern high-rise buildings have unique facades which partly rely on the incorporation of curtain walls. A curtain-wall system encloses the building to separate the internal and external environments. It can reduce building weight and it also transfers the wind load to the floor structure of the building. Wind-load codes govern the design of safe curtain wall systems against natural wind forces, considering direct static and dynamic pressure. In this paper aero-elastic considerations are investigated as a potential failure mode of curtain walls. Curtain-wall panels are regarded as comprising a flexible material such as glass and aluminium cladding subjected to an airflow that is parallel to their surface.
\end{abstract}

It is well-known that a flexible panel exposed to increasingly high flow speed will succumb to a divergence, or static buckling-type, instability at a particular critical flow speed. At a higher flow speed the panel will experience violent oscillatory flutter-type instability. Accordingly, we investigate the susceptibility of curtain-wall panels to aero-elastic effects.

A state-space model, based upon computational modelling, is used to investigate the aero-elastic stability of each flexible panel in isolation. We briefly present a recently developed approach and its new extension to theoretical modelling of the fully-coupled interaction between a simply-supported flexible panel and a fluid flow. We solve the boundary-value problem to determine the long-time response and investigate the effects on stability of adding localised structural inhomogeneity.

Localised structural inhomogeneity is incorporated as an additional single spring type support to the panel. The dependence of instability onset-flow speeds, and the forms of divergence and flutter instabilities, upon the added spring stiffness and its location are then investigated. Results show that the morphology of the unstable solution space significantly differs from that of the oft-studied corresponding hydro-elasticity problem because of the different density ratio between fluid and solid media. Of particular interest is that in the present aero-elastic system flutter occurs through the coalescence of two non-oscillatory unstable divergence modes.

The inclusion of a localised spring support to an otherwise unsupported panel is shown to be stabilising with respect to the critical divergence-onset flow speed and the limits to this strategy are identified. This strategy is marginally destabilising with respect to the more damaging flutter instability that occurs at higher wind speeds. However, at a sufficiently high spring-stiffness a sudden change to the solution morphology occurs that yields two unstable non-oscillatory divergence modes and flutter is postponed to much higher wind speeds. We close the paper with an assessment of what these results mean in dimensional terms as applied to different cladding panels. Overall, our results suggest a means to ameliorate adverse aero-elastic effects in potentially disastrous extreme wind-force situations such as those encountered in typhoons and tropical cyclones.

Keywords: aero-elasticity, curtain-walls, wind-load effects, buckling/flutter-type instability, computational modelling, state-space model 


\section{INTRODUCTION}

Fluid-structure Interactions (FSIs) feature the interaction of a fluid flow over a flexible plate. The plate could be part of a ship's hull, the skin of an aircraft fuselage or wing, window or roof of a building. The present paper is focused on the panels of an external curtain wall subjected to up to hurricane-force wind speeds aligned with their longitudinal axis; one such panel is schematically shown in Fig. 1. It is well known, e.g. Dugundji et al. (1963), Weaver \& Unny (1971), Ellen (1973), Lucey \& Carpenter (1993), Pitman \& Lucey (2009), that, for an initially flat plate, low-amplitude deformations generate a pressure field that, at critically high water or wind speeds, applies a force that amplifies the plate deformation. This is best known as the problem of panel flutter. There also exists a pre-flutter range of applied flow speeds in which a quasi-static or buckling-type of instability called divergence exists.

The vast majority of incompressible flow studies - in which the present regime of air-flow lies - have used water as the fluid medium. This gives a solid-to-fluid density ratio that is typically less than $10^{1}$. For airflow over a glass or aluminium panel, this ratio is more than $10^{3}$ giving a parameter regime that has not hitherto been explored presumably due to a lack of recognised applications until the emergence of curtain walls as an architectural feature. The present work shows that this parametric change leads to some surprising changes (from the now classical hydro-elastic case) in the morphology of the non-dimensional solution space.

In most engineered systems it is necessary to avoid panel instabilities, especially flutter because its motions are highly destructive in contrast to divergence instability that usually leads to a nonlinearly saturated statically deformed state of the panel. Designing out instability within the range of flow speeds to which the panel will be subjected is commonly achieved by material selection or increased use of material by way of a thicker plate, each of which effectively stiffens the flexible plate. However, these strategies are

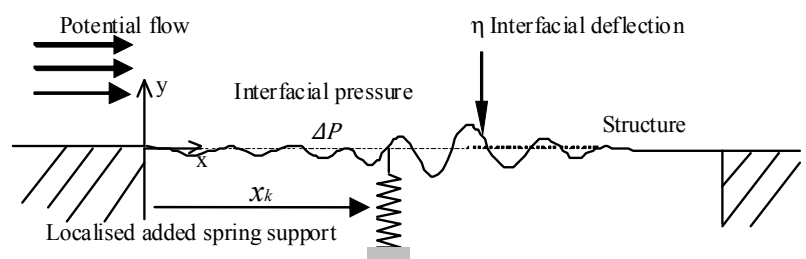

Figure 1: Schematic of a flexible panel in a uniform flow; the localised spring support is added as a stabilising strategy. costly both in financial terms and the penalty of increased dead-weight. In the present work we show how the inclusion of a highly localised spring support can be used to control the onset speed or even occurrence of divergence and flutter instabilities at flow- speeds within a desired operational range - in this case set by the maximum wind-speed in an extreme storm event.

\section{METHODS}

Our methods adopt the linear two-dimensional modelling of Pitman \& Lucey (2009), extending the structural side of the model to permit the inclusion of a localised spring support. Accordingly, only brief details are provided below, the reader being referred to that paper and Tan et al. (2010) for more detail.

The small-amplitude motion of a thin flexible plate, supported by an isolated spring at $x=x_{k}$ with stiffness constant $k$, in the presence of a fluid flow, Figure 1, is

$$
\rho_{m} h \frac{\partial^{2} \eta}{\partial t^{2}}+d \frac{\partial \eta}{\partial t}+B \frac{\partial^{4} \eta}{\partial x^{4}}+k \delta\left(x-x_{k}\right) \eta=-\Delta P(x, 0, t)
$$

where $\eta(x, t), \rho_{m}, h, d$ and $B$ are respectively, plate's deflection, density, thickness, damping coefficient and flexural rigidity of the flexible panel. The fluid pressure on the right-hand side, $\Delta P(x, y, t)$ is determined by solving the Laplace equation for the perturbation-velocity potential subject to the no-flux condition at the fluid-solid interface and decay to zero in the far field using a boundary-element method. The perturbationvelocity at the interface is then evaluated and used in the linearised unsteady Bernoulli equation following a streamline along $y=0$ from far upstream to determine the pressure along the interface. This yields the expression

$$
-\{\Delta P\}=2 \rho[\Phi]\left[D^{+}\right]\{\ddot{\eta}\}+2 \rho U_{\infty}[T]\left[D^{+}\right]\{\dot{\eta}\}+2 \rho U_{\infty}[\Phi]\left[D_{1}\right]\{\dot{\eta}\}+2 \rho U_{\infty}^{2}[T]\left[D_{1}\right]\{\eta\}
$$

where $[\Phi]$ and $[T]$ are $N x N$ matrices of invariant influence coefficients that arise from the boundary-element method deployed using $N$ panels of equal length to discretise the flexible surface, $\left[D^{+}\right]$and $\left[D_{l}\right]$ are spatially averaging and differentiation matrices, and $\rho$ and $U_{\infty}$ are the density and mean speed of the fluid; the over-dot notation on the interfacial variable indicates differentiation with respect to time. 
Equation (1) is written in finite-difference form, using the same discretisation that defined the boundaryelements in the flow solution and substitution of the expression for fluid pressure in Eqn. (2) yields the FSI system equation as

$$
[A]\{\ddot{\eta}\}+[B]\{\dot{\eta}\}+[C]\{\eta\}=0,
$$

where

$$
\begin{aligned}
& {[A]=-\rho_{m} h[I]+2 \rho[\Phi]\left[D^{+}\right],} \\
& {[B]=-d[I]+2 \rho U_{\infty}[T]\left[D^{+}\right]+2 \rho U_{\infty}[\Phi]\left[D_{1}\right],} \\
& {[C]=-B\left[D_{4}\right]-K\left[I^{k}\right]+2 \rho U_{\infty}{ }^{2}[T]\left[D_{1}\right],}
\end{aligned}
$$

and in which $[I]$ and $\left[D_{4}\right]$ are respectively, the identity and fourth-order spatial differentiation matrices. The matrix $\left[I^{k}\right]$ has zero entries everywhere except that the $i, i$ element is unity, where in the discretised system the $i^{t h}$ row corresponds to the location of the added spring at $x=x_{\mathrm{k}} . K$ is stiffness coefficient.

Introducing state variables $x_{i}=\eta_{i}$ and $x_{N+i}=\dot{\eta}_{i}$ for $\mathrm{i}$ from the $1^{\text {st }}$ point to the $N^{\text {th }}$ point, the $2 N$ output vector for the state-space model is $\{\{\eta\},\{\dot{\eta}\}\}^{T}=\{x\}$ and the state differential equation being expressed as

$$
\{\dot{x}\}=[H]\{x\}
$$

where $[H]=\left[\begin{array}{ll}0 & \mathrm{I} \\ F & E\end{array}\right]$ in which $[E]=-[A]^{-1}[B]$ and $[F]=-[A]^{-1}[C]$.

The long-time response is found by first assuming single-frequency response in the time domain, and then extracting the resulting eigenvalues of $[H]$. The single frequency response is proportional to $e^{s t}$ where $s$ is a complex variable. The system eigenvectors can then be used to assemble the deflection, $\eta(x, t)$, of the panel.

\section{RESULTS}

\subsection{The Effect of Density Ratio on System Stability}

We first present the FSI system dynamics in the context of airflow over a glass curtain-wall panel, showing how its behaviour differs from those of the more commonly studied applications in which the fluid is water. The dimensional properties used herein correspond to those of a purely elastic $(d=0)$ glass panel of length $L$ $=1.7 \mathrm{~m}$ with $h=4 \mathrm{~mm}, \rho_{\mathrm{m}}=2400 \mathrm{~kg} / \mathrm{m}^{3}$ and $B=381 \mathrm{Nm}$; the fluid is air with density $\rho=1.27 \mathrm{~kg} / \mathrm{m}^{3}$. Hinged-end restraints are applied to the panel. However, for scalability we present our results in nondimensional form. Non-dimensionalisation based upon panel length, $L$, and free-stream flow speed $U_{\infty}$ yields a system with two control parameters,

$$
\Lambda^{F}=\rho \frac{U_{\infty}{ }^{2} L^{3}}{B} \quad \text { and } \quad \mu=\frac{\rho_{m} h}{\rho L},
$$

described respectively as the stiffness and mass ratios; for a panel of given dimensions and material properties, the former represents the flow speed and the latter the density ratio $\left(\rho_{m} / \rho\right)$ of the two media. Figure 2 shows the variation of the first two system eigenvalues with stiffness ratio. Note that all of the system eigenvalues are calculated in our solution process but that our focus herein is upon the lowest frequency modes because these are the first to become unstable with increasing flow speed. The real and imaginary parts of the eigenvalues are respectively plotted in Figs. 2a and 2b. Positive(negative) real part indicates amplification(decay) of a mode while the imaginary part denotes the oscillation frequency of the panel. In addition to the results for air over glass $\left(\rho_{m} / \rho=2400 / 1.27\right)$, corresponding results for water over glass $\left(\rho_{m} / \rho=2400 / 1000\right)$ and an intermediate value of density ratio $\left(\rho_{m} / \rho=2400 / 100\right)$ are presented in Fig. 2.

At zero stiffness ratio all modes are purely oscillatory and neutrally stable. When air is the fluid medium, the frequencies of the first two modes air are much higher than their corresponding values for water. This is to be expected because the added mass due to movement of water as the panel vibrates is greater by virtue of its higher density. Increasing the stiffness ratio from zero reduces the oscillation frequency of panel oscillations until a critical value $\left(\Lambda^{F}=40\right)$ is reached at which Mode 1 ceases to oscillate - this marks the onset of divergence instability. This critical value agrees well with previous studies, e.g. Weaver \& Unny (1971), Ellen (1973), Lucey \& Carpenter (1993), which were based upon Galerkin methods. The present study demonstrates that this critical value is independent of density ratio as would be expected given that inertial forces are absent exactly at divergence onset where the fluid and structural stiffnesses exactly balance each other. 


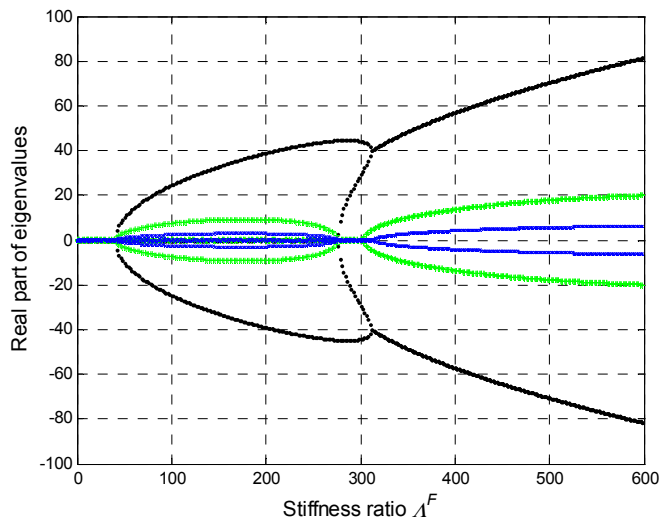

(a)

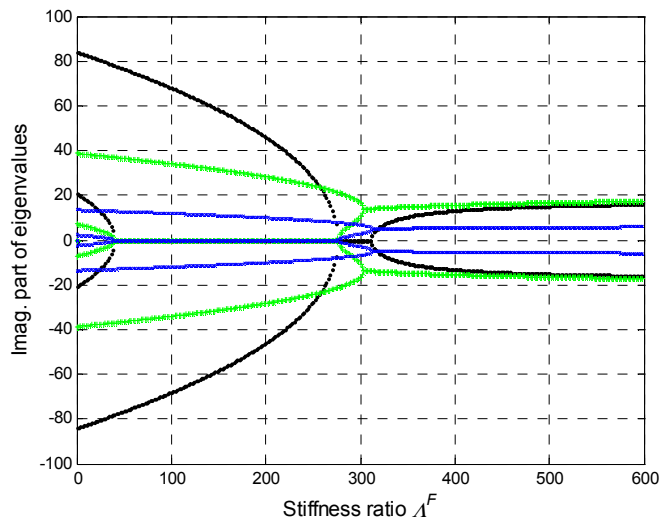

(b)

Figure 2: Variation of system eigenmodes with stiffness ratio (non-dimensional flow speed) for different values of density ratio $\left(\rho_{m} / \rho\right)$ : • (black), 2400/1.27; + (green), 2400/100; x (blue), 2400/1000 where (a) is the real (positive, growth; negative, decay) part, (b) is the imaginary (oscillatory) part of the eigenvalues.

At higher values of stiffness ratio, the panel experiences non-oscillatory divergence instability until a value of approximately 270 is reached. For the lower density ratios (including water flow), divergence recovery occurs whereas for airflow Mode 2 additionally succumbs to divergence instability. Continuing to increase the stiffness ratio yields a region of neutrally stable Mode 1 and Mode 2 oscillations for the lower density ratio cases followed by coalescence of these modes to give a powerful flutter instability. In contrast the airflow case features the coalescence of the two unstable non-oscillatory divergence Modes 1 and 2 to create the oscillatory flutter instability. This highly unusual (unique to the authors' knowledge) sequence of events in FSI - two non-oscillatory modes merging to give an oscillatory instability - occurs principally because for airflow the FSI is dominated by fluidstiffness effects due to the fourth term of the fluidpressure loading in Eqn. 2. For the equivalent fluid-

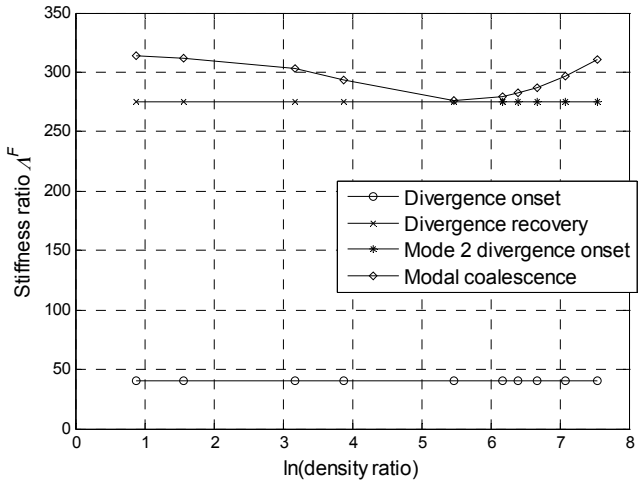

Figure 3: Variation of the stiffness ratio of critical points - divergence onset/recovery and modalcoalescence - in the eigenvalue space (typical of Fig. 2) with the solid-to-fluid density ratio. stiffness effects between air (with density $\rho_{\mathrm{A}}$ ) and water (with density $\rho_{\mathrm{W}}$ ), the fluid-damping and fluid inertia effects, respectively given by the third, second and first terms in Eqn. 2, are factors of $\left(\rho_{\mathrm{A}} / \rho_{\mathrm{W}}\right)^{(1 / 2)},\left(\rho_{\mathrm{A}} /\right.$ $\left.\rho_{\mathrm{W}}\right)^{(1 / 2)}$ and $\left(\rho_{\mathrm{A}} / \rho_{\mathrm{W}}\right)$ smaller for air. It is these terms that are principally responsible for the coupling of Modes 1 and 2 that lead to divergence recovery when water is the fluid medium.

To close this sub-section we explore how the switch of solution morphology depends upon the solid-to-fluid density ratio. Figure 3 shows the variation with density ratio $\left(\rho_{m} / \rho\right)$ of the values of stiffness ratio for each of Mode-1 and Mode-2 divergence-onset (where the latter occurs), divergence recovery and modal-coalescence flutter. It is clearly seen that the system solution follows the pattern typical of water over a flexible panel for density-ratio values up to a threshold value of approximately 240 (noting that $\ln (240)=5.48$ on the horizontal scale). For higher values than this, the fluid density is sufficiently low, relative to that of the solid, that both Modes 1 and 2 can concurrently succumb to divergence instability and then coalesce at higher flow speeds to create the flutter instability. We remark that in practical applications, only the Mode-1 divergence would be evident because it has a higher amplification rate than Mode 2 as can be seen in Fig. 2a.

\subsection{Stabilization using an Added Spring Support}

We now assess the effect of adding a single (line) spring support (see Fig. 1) to the glass panel as a strategy for controlling its aeroelastic destabilisation in high wind speeds; Tan et al. (2011) shows that this strategy works, at least conceptually, for marine applications. We continue with the same glass and air data listed in 
Section 3.1 but now adding a spring with stiffness constant varied in the form of multiples of $k$ where $k=$ $8500 \mathrm{~N} / \mathrm{m}^{2}$.

Figure 4 shows the variation with stiffness ratio (non-dimensional flow speed) of the system eigenvalues when the spring support is included at the panel mid-point for the cases $0 k$ (no spring, giving the result for $\rho_{m} / \rho=2400 / 1.27$ in Fig. 2), $1 k$ and $2 k$. As the spring constant, $k$, is increased, the critical value of $\Lambda^{F}$ set by the Mode-1 divergence-onset flow speed for a panel of given flexural rigidity, is seen to increase. This is readily understood by consideration of the mode shape of the instability. Mode 1 is principally the fundamental mode of panel vibration and thus placing the spring support at its anti-node clearly serves to suppress this mode. Figure 2 also shows the Mode- 2 divergence-onset at $\Lambda^{F} \approx 270$ is unaffected; this is because the spring is located at the node of the mode shape. The modal-coalescence flutter that occurs at $\Lambda^{F}$-values higher than those of Mode-1 and Mode-2 divergence onset is reduced to slightly lower flow speeds. The overall finding here is that adding a spring support is a very effective way to postpone the onset of aeroelastic buckling of the fundamental mode to higher airspeeds.

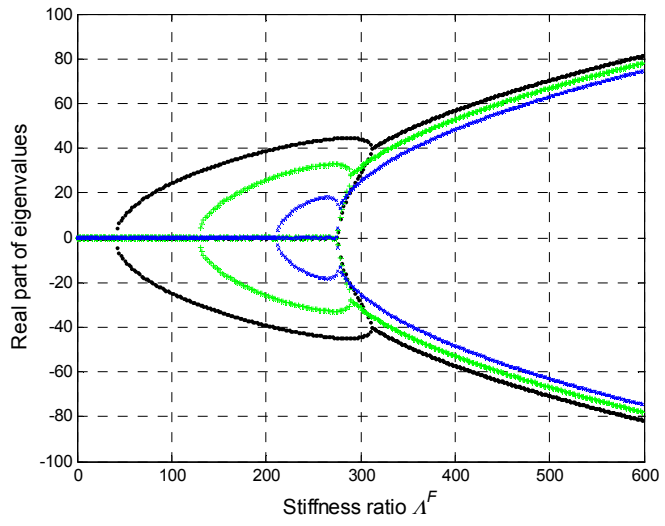

(a)

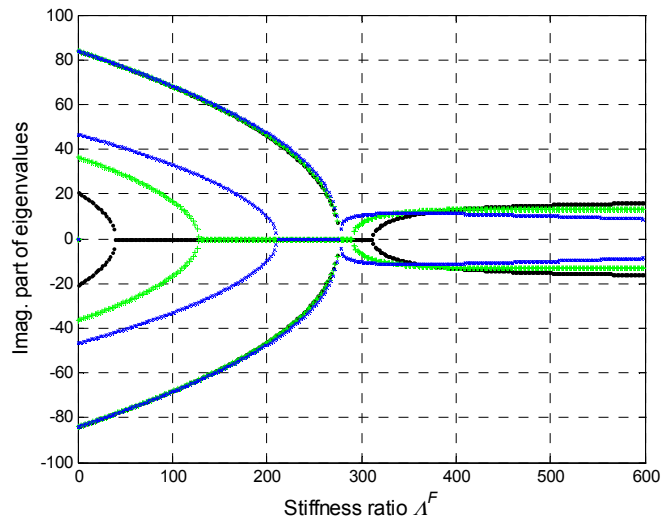

(b)

Figure 4: Variation of system eigenmodes with stiffness ratio (non-dimensional flow speed) for different values of localized spring support applied at panel mid-point: • (black), none; + (green), $1 k$; x (blue), $2 k$ where (a) is the real (positive, growth; negative, decay) part, and (b) is the imaginary (oscillatory) part of the eigenvalues.
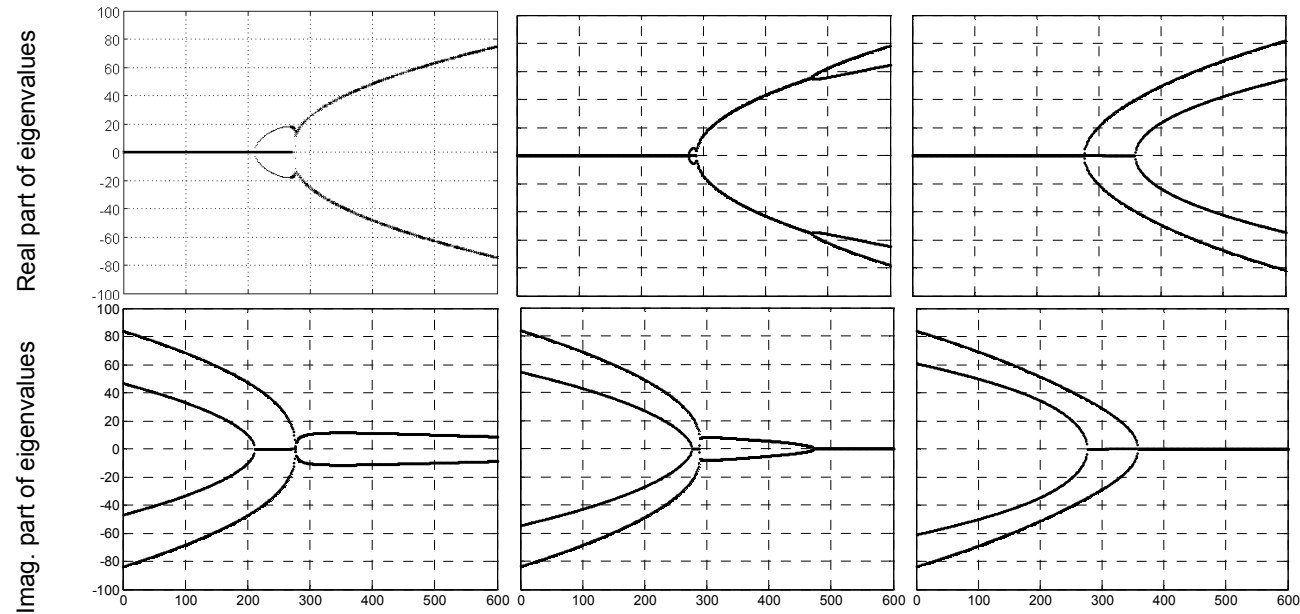

Stiffness ratio $\Lambda^{\mathrm{F}}$

Figure 5: Variation of system eigenmodes with stiffness ratio (non-dimensional flow speed) for different values of localized spring support applied at panel mid-point: the upper row represents the real part, the lower row the imaginary part and the first, second and third columns are respectively for $2 k, 3 k$, and $4 k$.

While the results of Fig. 4 demonstrate the control of divergence instability, the range of $k$ used therein has very little effect on the more destructive flutter instability at higher flow speeds. Figure 5 shows the effect of further increases to the stiffness of the spring support on the eigenvalue-solution morphology. The results for 
$2 k$ (first column) are replicated from Fig. 4 showing the standard airflow-over-glass sequence of instabilities. For $3 k$ the critical speeds of Mode- 1 and Mode- 2 divergence onset are almost identical; the significant feature here is that although modal-coalescence then occurs, its instability loop closes at $\Lambda^{F} \approx 460$ from thereon to be replaced by non-oscillatory divergence. A further increase to $4 k$ is seen to eliminate flutter and for it to be entirely replaced by both Mode- 1 and Mode- 2 divergence. Thus the inclusion of an isolated spring can be used to replace a more damaging flutter instability with buckling of the panel that would become static due to nonlinear saturation if some structural damping existed (Lucey et al. 1997).

We now summarize quantitatively the effects on the critical flow speeds for Mode- 1 and Mode-2 divergenceonset and flutter-onset speed (where these occur) of the stiffness of an added single spring support added at each of the panel mid-point and at $0.25 \mathrm{~L}$ from the leading edge of the glass panel. To do this, we change the non-dimensionalisation scheme from that of Eqn. (5) which was based upon panel length. The foregoing results show that for high stiffness the added spring can effectively shorten the panel length thus rendering this non-dimensional scheme inappropriate as a measure of divergence-onset flow speed. Thus, we allow panel length to be a free parameter and non-dimensionalise using the scheme presented in Lucey et al. (2003). This gives two new non-dimensional parameters,

$$
\bar{U}=U_{\infty} \frac{\left(\rho_{m} h\right)^{3 / 2}}{\rho B^{1 / 2}} \quad \text { and } \quad \bar{L}=\frac{L}{L_{r e f}},
$$

where the natural length scale is $L_{r e f}=\rho_{m} h / \rho$. Clearly their relationship to the non-dimensional stiffness ratio is $\Lambda^{F}=\bar{U}^{2} \bar{L}^{3}$. To complete the non-dimensional scheme, the coefficient of the added spring support is non-dimensionalised using $L_{\text {ref }}$ and the flexural rigidity of the plate. For a single localized spring support respectively, we then have $\bar{k}=k L_{r e f}{ }^{3} / B$. Thus, the non-dimensional critical speeds are functionally given as

$$
\bar{U}_{C}=f\left(\bar{L}, \bar{k}, \bar{x}_{k}\right)
$$

where $\bar{x}_{k}=x_{k} / L$ is the non-dimensional distance of the location of the added spring from the panel leading edge.

Figs. 6(a) and 6(b) show the variation of critical flow speeds with the magnitude of the added spring support for the two cases of spring location $\bar{x}_{k}=0.5$ and 0.25 ; in each figure $\bar{L}=0.2259$ (that gives the same physical length as the panels investigated through Figs. 2-5. These results clearly show that the addition of a single localized spring support can significantly increase the Mode 1divergence-onset flow speed. As could be expected on physical grounds, this strategy is more effective when the spring is placed at the panel midpoint. When placing it here, it is noted that there is a threshold of approximately $\bar{k}=2.69 \times 10^{4}$, for which the morphology of the solution changes, as shown through Fig. 5, and the modal-coalescence flutter of Modes 1 and 2 ceases to occur with an almost constant critical speed for Mode-1 divergence onset thereafter.

\section{SUMMARY}

A model fusing computational and theoretical methods, exploiting the advantages of each, has been used to predict the aero-behaviour of flexible panels. A particular merit of the approach is that it can be used to find the FSI eigenmodes of flexible panels and walls that include localized inhomogeneity.

The results presented herein for a purely elastic glass panel show that the solution morphology is significantly different from that of more dense fluids (e.g. water) interacting with flexible plate. Of particular note is that the present system features a range of flow speeds in with both Mode 1 and Mode 2 are unstable in divergence and that these non-oscillatory modes can coalesce with an increase in flow speed to create flutter instability. We have also shown that the addition of an isolated spring support to the panel can yield a very significant extension to the flow-speed range before divergence instability sets in and that further increases to its stiffness can cause the modal-coalescence flutter to be replaced by divergence although with no further increase in its critical speed.

To give an engineering feel for these benefits, we provide the following dimensional examples for divergence-instability onset. For a glass panel subjected to air flow with a single spring added at its midpoint, the critical-speed function in Eqn. 7 becomes $U_{C}=f(L / h, k, 0.5)$. Table 1 then lists the predicted divergence-onset flow speeds for each of three typical flat tempered glass panels for different values of the added spring support. Overall, these data show how the spring support can increase the allowable wind speed for the panels of curtain walls. 


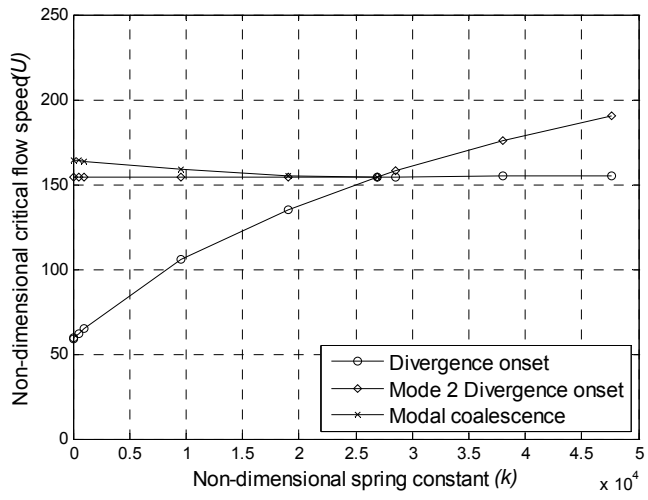

(a)

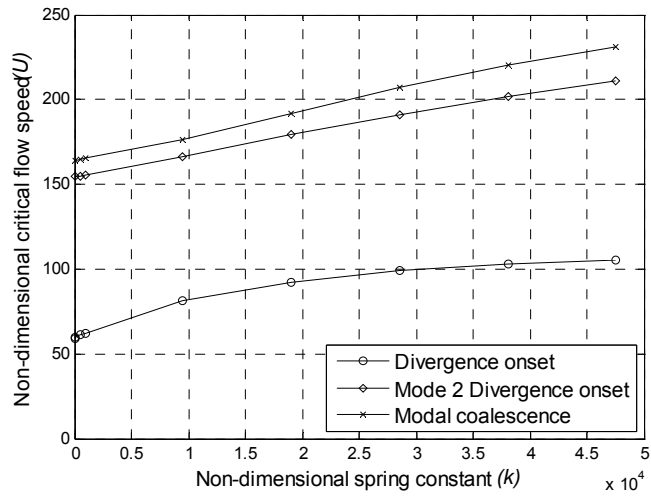

(b)

Figure 6: Variation of Mode-1 and Mode-2 divergence-onset, and modal-coalescence flutter-onset flow speeds with the coefficient of the added spring support for a panel with length $\bar{L}=0.2259$ : spring added at (a) panel mid-point, and (b) $0.25 \mathrm{~L}$ from the leading edge of the panel.

Table 1: Dimensional divergence-onset flow speeds $(\mathrm{km} / \mathrm{hr})$ for three typical flat tempered glass panels and with different levels of spring support.

\begin{tabular}{|c|c|c|c|}
\cline { 2 - 4 } \multicolumn{1}{c|}{} & \multicolumn{3}{c|}{$L / h$} \\
\hline $\begin{array}{c}\text { Spring constant } \\
\left(k=8500 \mathrm{~N} / \mathrm{m}^{2}\right)\end{array}$ & $\begin{array}{c}533 \\
(\mathrm{~L}=2.135 \mathrm{~m}, \mathrm{~h}=4 \mathrm{~mm})\end{array}$ & $\begin{array}{c}406 \\
(\mathrm{~L}=2.440 \mathrm{~m}, \mathrm{~h}=6 \mathrm{~mm})\end{array}$ & $\begin{array}{c}366 \\
(\mathrm{~L}=3.66 \mathrm{~m}, \mathrm{~h}=10 \mathrm{~mm})\end{array}$ \\
\hline $0 k$ & 126 & 189 & 219 \\
\hline $0.05 k$ & 139 & 200 & 230 \\
\hline $0.5 k$ & 224 & 266 & 287 \\
\hline
\end{tabular}

\section{ACKNOWLEDGEMENTS}

The authors acknowledge the support of the Australian Research Council through grant DP1096376.

\section{REFERENCES}

Dugundji, J., E. Dowell and B. Perkin (1963). Subsonic flutter of panels on a continuous elastic foundation. AIAA Journal 1, pp. 1146-1154.

Ellen, C.H. (1973). The stability of simply supported rectangular surfaces in uniform subsonic flow. ASME: Journal of Applied Mechanics 95, pp 68-72.

Lucey, A.D. and P.W. Carpenter (1993). The hydroelastic stability of three-dimensional disturbances of a finite compliant panel. Journal of Sound and Vibration 165, pp.527-552.

Lucey, A.D., G.J. Cafolla, P.W. Carpenter, and M. Yang (1997). The nonlinear hydroelastic behaviour of flexible walls. Journal of Fluids and Structures 11, pp. 717-744.

Lucey, A.D., P.K. Sen, and P.W. Carpenter (2003). Excitation and evolution of waves on an inhomogeneous flexible wall in a mean flow. Journal of Fluids and Structures 18, pp. 251-267.

Pitman, M.W. and A.D. Lucey (2009). On the direct determination of the eigenmodes of finite flow-structure systems. Proceedings of the Royal Society A 465, 257-281.

Tan B.H., A.D. Lucey, and M.W. Pitman (2010). Hydroelastic stability of flexible panel: eigen-analysis and time-domain response. Proceedings of ASME $20103^{\text {rd }}$ Joint US-European Fluids Engineering Summer Meeting, Montreal, Canada, Paper no. FEDSM-ICNMM2010-30057.

Tan B.H., A.D. Lucey, and M.W. Pitman (2011). Controlling hydroelastic instability of hull panels through structural inhomogeneity. In: RINA, Royal Institution of Naval Architects - International Conference, High Speed Marine Vessels, 2-3 March 2011, Fremantle, pp. 51-60

Weaver, D.S. and T.S. Unny (1971). The hydroelastic stability of a flat plate. ASME: Journal of Applied Mechanics 37, pp 823-827. 